\title{
Low Tension Glaucoma - Is Treatment Worthwhile?
}

\author{
ROGER A. HITCHINGS \\ London
}

Low tension glaucoma (LTG) may be considered to be a variety of primary open angle glaucoma where glaucomatous cupping and typical field loss coexist, but without raised intraocular pressure (IOP). These patients have open angles and do not have other cause for the cupping and field loss. ${ }^{1}$ The diagnosis should be restricted to patients who, over a 24 hour period have a peak IOP of $<24 \mathrm{~mm} \mathrm{hg}$. and a mean IOP below $21 \mathrm{~mm}$ hg. Such individuals should have a neurological examination to exclude a retroocular cause for the visual loss. Thus defined, patients with low tension glaucoma typically have bilateral disease. The male/female ratio is $1: 2$. In addition they frequently have a history of classical migraine. The prevalence of low tension glaucoma appears to be low, in a general glaucoma clinic they will probably account for less than $10 \%$ of all patients with primary open angle glaucoma.

Opinions about the physical signs differ, the optic disc has been thought by some, ${ }^{2}$ but not others ${ }^{3}$ to have a different appearance from that seen in primary open angle glaucoma. Similarly, differences in the visual fields have been noted by some ${ }^{4,5}$ although no differences have been found by others. ${ }^{3,6,7}$

The pathogenesis of low tension glaucoma is obscure. A vascular hypothesis has been favoured for many years and systemic factors such as cardiovascular disease, hypertension, shock from sudden blood loss and a sedentary life style have all been implicated ${ }^{8-10}$ In addition it has been suggested that biochemical factors may be important. ${ }^{11,12} \mathrm{By}$ contrast, there is a strong argument for a mechanistic hypothesis. ${ }^{13}$

The natural history of low tension glaucoma is ill understood. Chumbley and Brubaker ${ }^{14}$ reviewed 34 eyes of 17 patients with more than one visual field examination and who had been followed for 4-12 years. They found that $41 \%$ of these patients showed progression. Levene ${ }^{1}$ also noted progression in $40 \%$ of his series of 34 eyes in 23 cases. In a recent review of patients with low tension glaucoma we looked at the patients attending the Glaucoma Clinic at Moorfields Eye Hospital. ${ }^{15}$ The Goldmann field charts of fifty six patients who had been followed for a minimum of 3.5 years and a mean of 10 years were reviewed. Each patient had had an average of 1.5 fields per year. For the purpose of the study each visual field was divided into an upper and lower half and the percentage of seeing field to an I-4 target in each half was found for each visit $(47.5 \%$ of the total per hemifield as the blind spot accounted for $5 \%$ of the total). In this study we also found that only $40 \%$ progressed. When progression did occur it was more likely in an already damaged hemifield. We found that the rate of progression differed considerably from eye to eye and that it could be extremely erratic for any one eye.

From this retrospective study it appeared that with 'no progression' in $60 \%$ of eyes, despite many years of follow-up, it was unreasonable to start treating patients with low tension glaucoma as soon as the diagnosis was made. A more appropriate method was to see if 'progression' occurred. Statistical analysis of the fields in this study showed that to achieve a $95 \%$ confidence limit for identifying 'progression' a sequence of at least four, or preferably five, fields 
needed to be obtained. ${ }^{16}$ Similar conclusions were reached by Schwartz and colleagues with Trend Analysis. ${ }^{17}$ (We used a single isopter and kinetic perimetry for this purpose. Modern computer assisted perimetry could be expected to provide earlier notice of change in the visual field although the same sequence of 4-5 fields is required. ${ }^{17}$ )

Knowing that a significant proportion of patients with low tension glaucoma do progress, but erratically; deciding on effective treatment becomes a problem. There does not seem to be any method of identifying those patients who will progress before they actually do so. At the present time the only therapeutic approach is hypotensive therapy. To achieve a clinically significant fall in IOP in these patients (at least $5 \mathrm{~mm} \mathrm{hg.)} \mathrm{is} \mathrm{a} \mathrm{major} \mathrm{challenge.} \mathrm{We} \mathrm{felt}$ that fistulising surgery would be the most direct method and could be done on one eye of patients with progressive disease. This study reports on the medium term results of fistulising surgery on a small group of such patients.

\section{Materials and Methods}

Patients with progressive low tension glaucoma and bilateral disease were offered a trabeculectomy to the 'worse' eye. (Progression was defined on the 95\% confidence limits outlined above using the I-4 target on the Goldmann Perimeter). The patients had the nature of their disease explained to them, together with the reason for recommending fistulising surgery. The possible advantages and disadvantages of fistulising surgery were explained. Those patients who agreed to undergo surgery form the basis of this report.

Patients undergoing surgery had regular follow-up examinations, these included checks on their IOP and (Humphrey) visual fields. The mean of the last three pre-trial IOPs for each eye was compared with the IOPs found during the follow-up period. Using the Humphrey Perimeter the 30-2 program was performed with the size 3 target (each patient was 'trained' to undergo this test by having at least one preliminary 'dry run' beforehand). 'Statpac' analysis was carried out on the visual fields, together with a comparison of the individual retinal point sensitivity for the two eyes.

\section{Results}

To date six patients with progressive low tension glaucoma have been studied. They have been followed for 15 to 21 months after undergoing surgery.

The mean preoperative IOP in the eyes undergoing surgery was $15.5 \mathrm{~mm} \mathrm{Hg}$., for the eyes not undergoing surgery the IOP was $14.8 \mathrm{~mm} \mathrm{Hg}$.

The follow-up IOPs have been set out in the Table. The operated eye consistently had the lower IOP in five of the six patients. The difference between the two eyes ranged between 0 and $6.3 \mathrm{~mm} \mathrm{Hg}$.

Sequential visual field changes, together with statpac analysis for one patient have been shown (for the purpose of illustration) in the figure. It will be seen that no difference is apparent in the rate of visual field loss between the two eyes. The other 5 patients showed a similar lack of progression.

\section{Discussion}

This report has looked at the rationale for treating patients with low tension glaucoma. Previous studies have shown that any patient newly diagnosed as having this condition will have a $60 / 40$ chance of not progressing over a 10 year period..$^{15}$ In addition, in those eyes where progression does occur, the rate varies from eye to eye and, for any one eye, may vary over time. ${ }^{15}$ Because of these observations it is suggested that treatment be witheld until progression is certain.

This study and one previous study ${ }^{18}$ have

Table I Comparison IOP levels in operated and non-operated eye

\begin{tabular}{|c|c|c|c|c|c|}
\hline & \multicolumn{2}{|c|}{$\begin{array}{l}\text { Operated eye } \\
\text { mean IOP }\end{array}$} & \multicolumn{3}{|c|}{$\begin{array}{l}\text { Non-operated eye } \\
\text { mean IOP }\end{array}$} \\
\hline & $\mathrm{mm} \mathrm{Hg}$ & $(S D)$ & $\mathrm{mm} \mathrm{Hg}$ & $(S D)$ & $P$ value \\
\hline 1. & 11.5 & $(2.05)$ & 16.5 & $(1.0)$ & $<0.01$ \\
\hline 2. & 13.25 & $(2.05)$ & 17.25 & $(2.5)$ & $<0.01$ \\
\hline 3. & 6.25 & $(2.87)$ & 13.5 & (1.91) & $<0.05$ \\
\hline 4. & 11.00 & (1.22) & 14.8 & (1.10) & $<0.001$ \\
\hline 5. & 16.3 & (2.29) & 16.3 & (1.79) & $>0.7$ \\
\hline 6. & 10.00 & $(2.65)$ & 16.33 & (2.39) & $<0.01$ \\
\hline
\end{tabular}



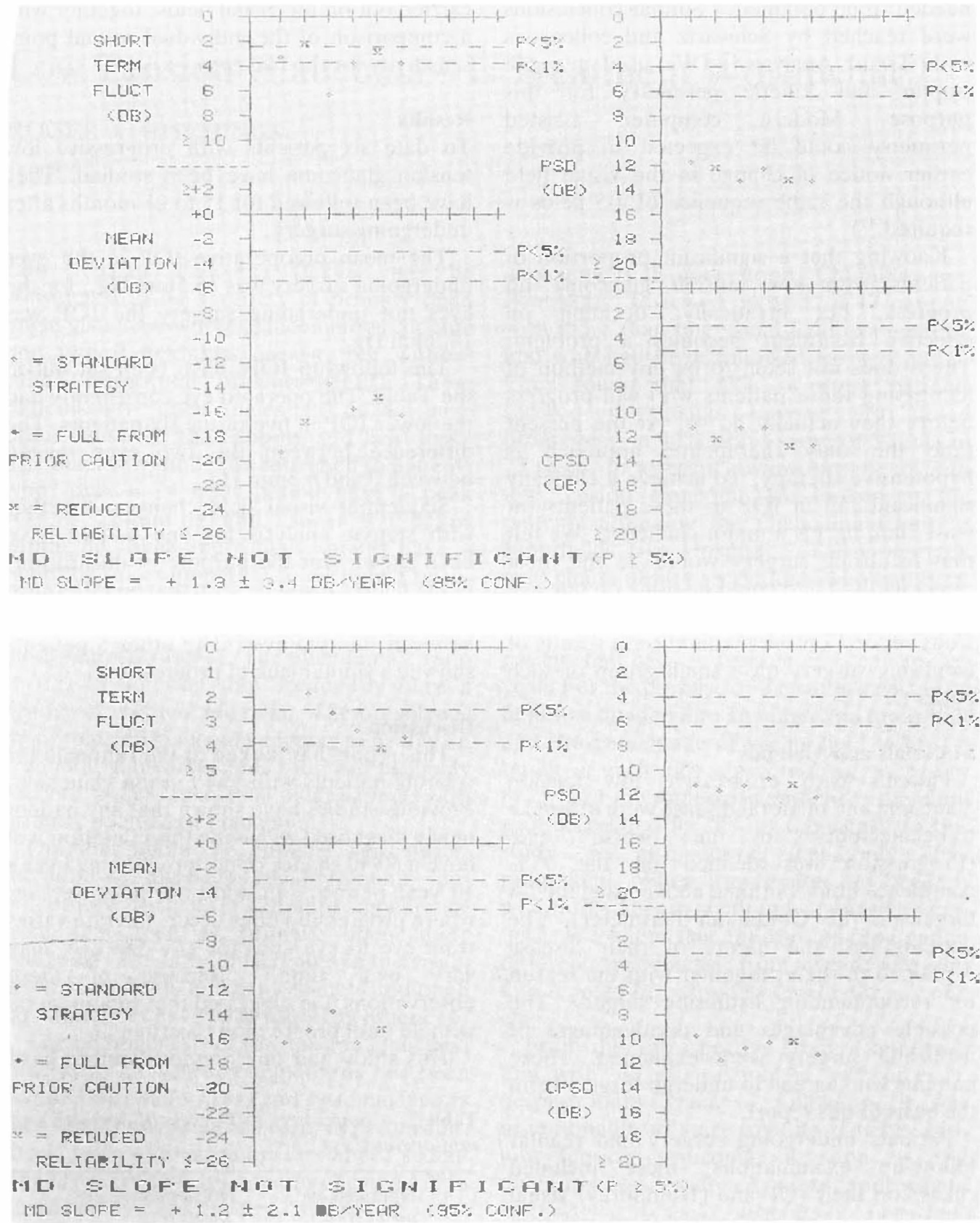

Fig. 1 The figure shows visual field results from one patient. The four graphs (from Statpac Analysis of results from a Humphrey Field Analyser) summarise the visual field plots over the follow-up period when 6 visual field examinations were performed.

The results are presented as global indices where they measure the performance of the whole of the visual field (see text for discussion of the merits of this approach).

Short term fluctuation is an expression of the patient's reproducability on re-testing of individual retinal spots. The "P" (probability) value shows the levels achieved by the normal population, i.e. $<5 \%$ of the normal age matched population will show a short term fluctuation $(S F)$ of $>2.25 D b$. 
noted how difficult it is to lower IOP by a significant amount in patients with low tension glaucoma. It had previously been the experience of the author that topically applied treatment rarely achieved this aim; in any one patient a combination of medical, laser and, probably, surgery would be required. The patients reported on here show that in the medium term fistulising surgery could not always produce a 'clinically significant' lowering of IOP. In five of the 6 patients significant lowering of IOP was achieved over the period of study. However, even after fistulising surgery, the hypotensive effect may be limited, perhaps by the formation of fibrous tissue around the sclerostomy. Modifying the operation by the use of a contact lens affixed after surgery together with systemic steroids has been reported to have good results. ${ }^{18}$ Even with this approach it should be remembered that episcleral venous pressure may play an important part in limiting the lowest achievable IOP after local drainage surgery. A technique which could overcome these problems would be to direct aqueous posteriorly, via a drainage tube in the anterior chamber, to a large draining area in the orbit. Such an approach has already been used in cases of 'difficult' glaucoma by means of a 'tube and gutter' operation.

The arguments outlined above indicate the size of the problems that need to be overcome when contemplating treatment in patients with low tension glaucoma. For these reasons it is suggested that treatment designed to lower IOP by a clinically significant amount should be limited to patients for whom undoubted progression has occurred and who are still youthful.

The six patients is this study underwent fistulising surgery to one eye. Five of the 6 eyes demonstrated a consistent lowering of
IOP in the operated eye. They were all followed for a minimum of 15 months. Humphrey 30-2 program perimetry was carried out at regular intervals on each patient. The patients were able to provide reliable fields. Despite achieving a persistent IOP difference between the two eyes in five of these patients, analysis of the Global Indices MD and CPSD on the Humphrey Statpac did not show any sign of continued progression. The follow-up period has, so far, been comparatively short. A longer period of follow-up might demonstrate differences with this method of analysis between the visual fields in any one patient. Alternatively, analysis of change occuring in sectors of the field, or change in the sensitivity of individual retinal points (Trend Analysis) ${ }^{17}$ may prove to be a more accurate approach. Until the time that such an analysis is carried out on these patients sequential analysis of the Global Indices offers the best method of following this type of patient.

The results presented here show that it is possible to obtain a persistent lowering of IOP in low tension glaucoma. For the occasional patient with progressive low tension glaucoma such aggressive therapy may be justified. On the available evidence, however, it is not yet proven that such treatment alters the rate of progression or that it is of benefit to the patient.

S. Poinoosawmy helped with data compilation and performed the visual field analyses.

\section{References}

${ }^{1}$ Levene RZ: Low tension glaucoma: A critical review and new material. Surv. Ophthalmol. 1980 24: 621-42.

2 Caprioli $\mathbf{J}$ and Spaeth GL: Comparison of the optic nerve head in high- and low-tension

The mean deviation is an expression of generalised depression of the visual field and, again, "P" values for the normal population have been shown.

PSD and CPSD (pattern standard deviation and corrected pattern standard deviation) are values for "undulation" or "focal depression" on the hill of vision. The PSD and CPSD summarise any such changes. The " $P$ " value for the age matched normal population is shown.

It will be seen that, for the two eyes of the patient illustrated, a) the results are quite abnormal and, $b$ ) they have not changed over the test period. This similarity between the two eyes occurs despite the presence of $a$ significantly different level of intra-ocular pressure maintained between the two eyes. 
glaucoma. Arch Ophthalmol 1985103 1145-9.

${ }^{3}$ Lewis RA, Hayreh SS, Phelps CD: Optic disc and visual field correlations in primary open angle glaucoma and low tension glaucoma. Am J Ophthalmol 1983; 96: 148-52.

${ }^{4}$ Caprioli J and Spaeth GL: Comparison of visual defects in low tension glaucoma with those in high tension glaucoma. Am J Ophthalmol 1984; 97: 730-7.

${ }^{5}$ Anderton S and Hitchings RA: A comparative study of the visual field of patients with low tension glaucoma and those with chronic simple glaucoma. Docum Ophthalmol Proc Ser 35. 5th International Visual Field Symposium, Sacromento 1982. ed Greve EL and Heijl A, DrW Junk pubs. pp97-99.

${ }^{6}$ Motolko M, Drance SM, Douglas GR: Visual field defects in low tension glaucoma. Comparison of defects in low tension glaucoma and chronic open angle glaucoma. Arch Ophthalmol 1982; 100: 1074-7.

${ }^{7}$ Greve EL and Geijssen HC: Comparison of glaucoma visual field defects in patients with high and low intraocular pressures. Doc Ophthalmol Proc Series 35. 5th International Visual Field Symposium, Sacramento 1982. ed Greve EL and Heijl A Dr W. junk pubs. 107-111.

${ }^{8}$ Drance SM, Morgan RW, Sweeney VP: Shock induced optic neuropathy. New Eng J Med 1973; 288: 392-5.

${ }^{9}$ Drance SM, Sweeney VP, Morgan RW, Goldman F: Factors involved in the production of low tension glaucoma. Can J Ophthalmol 1974; 9: 399-406.

${ }^{10}$ Goldberg I, Hollows FC, Cass MA, Becker B:
Systemic factors in patients with low tension glaucoma. Br J Ophthalmol 1981; 65: 56-62.

${ }^{11}$ Winder AF, Paterson J, Miller SJH: Biochemical abnormalities associated with ocular hypertension and low tension glaucoma. Trans Ophthalmol Soc UK 1974; 94: 518-24.

12 Klaver JHJ, Greve EJ, Gosling H, Geijssen HC, Heyvelmans JHA: Blood and plasma viscosity measurements in patients with glaucoma. Br J Ophthalmol 1985; 69: 765-70.

${ }^{13}$ Quigley HA, Addicks EM, Green WR, Morany AE: Optic nerve damage in human glaucoma2 . The site of injury and susceptibility to damage. Arch Ophthalmol 1987; 99: 635-49.

${ }^{14}$ Chumbley LC and Brubaker RF. Low tension glaucoma. Am J Ophthalmol 1976; 81: 761-5.

${ }^{15}$ Anderton S, Coakes R, Poinoosawmy S, Clarke $\mathrm{P}$, Hitchings RA: The nature of visual loss in low tension glaucoma. Doc Ophthalmol Proc Series 42. 6th International Visual Field Symposium, Santa Margherita Ligure 1984. Eds Heijl A and Greve E. pubs Dr W Junk. 383-6.

${ }^{16}$ Clarke P: Sequential analysis of visual fields, a statistical study. Clarke P (Unpublished observations).

${ }^{17}$ Wu D-C, Schwartz B, Nagin P: Trend analysis of automated visual fields. Docum Ophthalmol Proc Series 49. 7th International Visual Fields Symposium. Amsterdam 1968. Eds Greve EL and Heijl A. Dr W Junk pubs. 175-190.

18 Abedin S, Simmons RJ, Grant WM: Progressive low tension glaucoma. Ophthalmology 1982; 89: $1-6$. 